\title{
Bio-efficacy Studies of Quinalphos 25\% EC against Insect Pests of Red Gram
}

\author{
A. Vyas, M.K. Mahla, G. Chhangani* and N.L. Dangi \\ Department of Entomology, Rajasthan College of Agriculture (MPUAT) Udaipur, India \\ *Corresponding author
}

\section{A B S T R A C T}

\section{Keywords \\ Bio-efficacy, Quinalphos, Insect pests, Red gram}

\section{Article Info}

Accepted: 06 June 2018 Available Online: 10 July 2018
The investigation on, "Bio Efficacy of Quinalphos $25 \%$ EC Against Insect Pests of Red Gram" was conducted at Instructional Farm and Department of Entomology, Rajasthan College of Agriculture, Udaipur during kharif 2016 and 2017. The red gram variety "ICPL-87" was sown to record the different insect pests population and bioefficacy of quinalphos $25 \mathrm{EC}$ against the pod borer and pod fly population. Among the different treatments quinalphos $25 \mathrm{EC}$ had the lowest pest population in both the years. The treatment with quinalphos 25 EC @ $750 \mathrm{~g}$ a.i./ ha had the lowest pod borer population (2.08 larvae/ plant) after 10 days of spraying. Similarly, it had lowest pod fly damage (4.99\%). The same trend was observed during both the year 2016 \& 2017.

\section{Introduction}

Pulse is an integral part of human diet and fulfills the protein requirement of majority of the Indian population besides providing energy. They also supply certain essential amino - acids minerals and vitamins which are crucial for normal health. Nature has also endowed the pulses with a unique mechanism of nitrogen fixation which helps in sustaining soil fertility. The pigeonpea crop is grown as an annual crop in India and as a perennial in many other countries where the pods are harvested at regular intervals. About 90 percent of the world's pigeonpea is produced in India. Pigeonpea is the second important pulse crop of the country occupying 14.5 percent of the area and contributes 15.5 percent of the total pulse production. It is predominantly cultivated in Maharashtra, Uttar Pradesh, Madhya Pradesh, Karnataka, Gujarat and Andhra Pradesh. These states contribute about 86.8 percent of the area and 84.4 percent of the production (Panda, 2005). Biotic and abiotic factors are responsible for low yields of the crop. Among the biotic factors insect pests constitute one of the major factors affecting both production and yield stability of the crop. More than 200 insect pests and other arthropods are known to be 
associated with this crop (La I, 1981). The damage in the vegetative stage of the crop is of occasional importance but the damage, which occurs in the reproductive phase, affects the production and yield (Thakur, 1989). They are mainly pod infesting species i.e. gram pod borer, Helicoverpa armigera (Hub.) 1 (Lepidoptera: Noctuidae); pigeonpea pod fly, Melanagromyza obtusa (Malloch) (Diptera: Agromyzidae); pigeonpea pod bug, Cavigralla gibbosa Spin. (Hemiptera: Coreidae); pigeonpea plume moth, Exelastis atomosa W. (Lepidoptera: Pterophoridae) and pulse beetle, Callosobruchus sp. which are more or less uniformly distributed in Madhya Pradesh. The pest complex cause adequate economic damage leading to very low yield levels of $500-800 \mathrm{~kg} / \mathrm{ha}$ as against the potential yields of 1800 to $2000 \mathrm{~kg}$ / ha (La et al., 1997). The pigeonpea pod borer, Helicoverpa armigera is the foremost among the insect pests of pigeonpea that causes serious damage to the crop (Pawar and Shalla, 1975; Singh and Singh 1978; Sithanantham et al., 1987; Singh and Singh, 1991; Sanap et al., 1995; Prasad, 1997; Bhubaneshwari and Balasuranathan, 2002; Sunil Kumar et al., 2003; Akhilesh Kumar, 2004). The larva damages the buds, flowers, pods and developing seeds (Sithanantham et al., 1987). In recent years, management of $H$. armigera has become difficult due to development of resistance is all the major insecticide classes available to Indian farmers (MeCafferry et al., 1989 and Armes et al., 1992), Hence, there is an urgent need to develop alternative control methods, which should be made available to the pigeonpea cultivators. In this context, studies on bioefficacy of Quinalphos $25 \%$ EC will be highly remarkable in the management of different insect pests of Red gram.

\section{Materials and Methods}

The bio-efficacy of Quinalphos 25\% EC at $325,350,375$ and $750 \mathrm{~g}$ a.i./ha, Indoxacarb
$14.5 \% \mathrm{SC}$ at $60 \mathrm{~g}$ a.i./ha and Deltamethrin $2.8 \% \mathrm{EC}$ at $12.5 \mathrm{~g}$ a.i./ha were evaluated against pod borer, pod fly, aphids, jassids, thrips and mites in red gram during Kharif 2016 and 2017. The experiment was conducted in randomized block design with seven treatments replicated three times at the instructional Farm, R.C.A., Udaipur. The red gram variety ICPL-87 was shown on $29^{\text {th }}$ and $27^{\text {th }}$ August, during Kharif 2016 and Kharif 2017. Each treatment was applied three times. The observation on the population of aphids, jassids, white fly thrips and mites were recorded on three top and two middle leaves per plant (5 leaves/plant) on five plants selected randomly while pod borer in 5 plants and per cent pod damage by pod borer and pod fly. The observation was recorded one day before and at one, three, five, seven and ten days after each spray and mean reduction in population was calculated at 1, 3, 5, 7 and 10 days after each sprays.

The pretreatment population of insect pests namely pod borer, pod fly, Aphids, Jassids, Whiteflies, Thrips, mites were recorded 24 hours before spray on randomly selected five plants in each replication.

\section{Results and Discussion}

\section{Bio-efficacy}

\section{Effect of Quinalphos 25\% EC against population of $H$. armigera}

During Kharif, 2016 and 2017 the average population of $H$. armigera before sprays ranged from 6.74 to 7.37 and 6.13 to 6.76 per 5 plants (randomly selected), respectively. At 1, 3, 5, 7 and 10 days after all three spray, the larval population per 5 plants was found significantly lowest in the treatments of Quinalphos 25\% EC. At final observation i.e. 10 days after third application, minimum $H$. armigera population per 5 plants was recorded 
in the treatment of Quinalphos 25\% EC @ 750 g a.i./ha (2.08) followed by Quinalphos 25\% EC @ $375 \mathrm{~g}$ a.i./ha (3.18), which was at par with Quinalphos 25\% EC @ $350 \mathrm{~g}$ a.i./ha (3.24), followed by Indoxacarb 14.5\% SC (3.28), Quinalphos 25\% EC @ $325 \mathrm{~g}$ a.i./ha (3.70) and Deltamethrin 2.8\% EC (4.30) (Table 1). The maximum $H$. armigera larval count was recorded in untreated control i.e. 9.00. The similar trend was followed in 2017 (Table 2).

Effect of different doses of Quinalphos 25\% EC on per cent pod damage by $\boldsymbol{H}$. armigera and M. obtusa during 2016 and 2017

During Kharif 2016 and 2017, the per cent pod damage by $H$. armigera ranged between 4.99 to 10.96 per cent and 6.97 to 12.94 per cent respectively (Table 3). During Kharif 2016 the least per cent pod damage was found in the treatment comprising of Quinalphos
25\% EC @ 750 g a.i./ha (4.99 per cent), followed by the treatment of Quinalphos $25 \%$ EC @ $375 \mathrm{~g}$ a.i./ha with 6.91 per cent which was statistically at par with Quinalphos $25 \%$ EC @ $350 \mathrm{~g}$ a.i./ha with 7.20 per cent pod damage. The highest per cent pod damage by pod borer was found in the untreated control. The similar trend followed in 2017.

The per cent pod damage by $M$. obtusa ranged from 33.04 per cent to 40.39 per cent (2016) and 37.34 to 43.69 per cent (2017) (Table 4) among the treatments. The highest percent pod damage was in the untreated control. The treatment comprising of Quinalphos 25\% EC @ $750 \mathrm{~g}$ a.i./ha (33.04 per cent) showed least per cent pod damage, followed by the treatment of Quinalphos 25\% EC @ $375 \mathrm{~g}$ a.i./ha with 33.41 per cent pod damage and it was at par with Quinalphos 25\% EC @ $350 \mathrm{~g}$ a.i./ha with 33.83 per cent pod damage. The same trend followed in 2017.

Table.1a Treatment Details: Seven treatments (including control)

\begin{tabular}{|c|l|c|c|}
\hline S. No. & Treatments & \multicolumn{2}{|c|}{ Dosage } \\
\cline { 3 - 4 } & & g. a.i./ha & $\mathrm{ml} / \mathrm{ha}$ \\
\hline $\mathbf{1 .}$ & Quinalphos 25\% EC & 325 & 1300 \\
\hline $\mathbf{2 .}$ & Quinalphos 25\% EC & 350 & 1400 \\
\hline $\mathbf{3 .}$ & Quinalphos 25\% EC & 375 & 1500 \\
\hline $\mathbf{4 .}$ & Quinalphos 25\% EC & 750 & 3000 \\
\hline $\mathbf{5 .}$ & Indoxacarb 14.5\% SC & 60 & 400 \\
\hline $\mathbf{6 .}$ & Deltamethrin 2.8\% EC & 12.5 & 500 \\
\hline $\mathbf{7 .}$ & Untreated control & - & - \\
\hline
\end{tabular}

Table.1b Number and date of spray: Three

\begin{tabular}{|c|l|l|l|}
\hline S. No. & Spray & \multicolumn{1}{|c|}{ Kharif 2016 } & \multicolumn{1}{c|}{ Kharif 2017 } \\
\hline 1. & First & 29 September 2016 & 22 October 2017 \\
\hline 2. & Second & 20 October 2016 & 01 November 2017 \\
\hline 3. & Third & 07 November 2016 & 11 November 2017 \\
\hline
\end{tabular}


Table.1 Bio-efficacy of Quinalphos 25\% EC against H. armigera on red gram during Kharif, 2016

\begin{tabular}{|c|c|c|c|c|c|c|c|c|c|c|c|c|c|c|c|c|c|}
\hline \multirow{3}{*}{ Treatments } & \multirow{3}{*}{$\begin{array}{c}\text { Dose } \\
\text { (g a.i./ha) }\end{array}$} & \multirow{3}{*}{ PTC } & \multicolumn{15}{|c|}{ Average no. of larval population / 5 Plants } \\
\hline & & & \multicolumn{5}{|c|}{ First Spray } & \multicolumn{5}{|c|}{ Second spray } & \multicolumn{5}{|c|}{ Third spray } \\
\hline & & & 1 DAS & $\begin{array}{c}3 \\
\text { DAS }\end{array}$ & 5 DAS & 7 DAS & $\begin{array}{c}10 \\
\text { DAS }\end{array}$ & 1 DAS & 3 DAS & 5 DAS & 7 DAS & $\begin{array}{c}10 \\
\text { DAS }\end{array}$ & 1 DAS & 3 DAS & 5 DAS & $\begin{array}{c}7 \\
\text { DAS }\end{array}$ & $\begin{array}{c}10 \\
\text { DAS }\end{array}$ \\
\hline Quinalphos 25\% EC & 325 & $\begin{array}{c}6.93 \\
(2.62)\end{array}$ & $\begin{array}{c}6.77 \\
(2.59)\end{array}$ & $\begin{array}{c}6.68 \\
(2.53)\end{array}$ & $\begin{array}{c}6.44 \\
(2.51)\end{array}$ & $\begin{array}{c}6.59 \\
(2.56)\end{array}$ & $\begin{array}{c}6.55 \\
(2.57)\end{array}$ & $\begin{array}{c}6.32 \\
(2.50)\end{array}$ & $\begin{array}{c}6.14 \\
(2.46)\end{array}$ & $\begin{array}{c}6.05 \\
(2.44)\end{array}$ & $\begin{array}{c}5.33 \\
(2.82)\end{array}$ & $\begin{array}{c}5.41 \\
(2.30)\end{array}$ & $\begin{array}{c}5.18 \\
(2.25)\end{array}$ & $\begin{array}{c}4.75 \\
(2.14)\end{array}$ & $\begin{array}{c}4.30 \\
(2.03)\end{array}$ & $\begin{array}{c}3.95 \\
(1.94)\end{array}$ & $\begin{array}{c}3.70 \\
(1.87)\end{array}$ \\
\hline Quinalphos 25\% EC & 350 & $\begin{array}{c}6.74 \\
(2.59)\end{array}$ & $\begin{array}{c}6.68 \\
(2.57)\end{array}$ & $\begin{array}{c}5.48 \\
(2.32)\end{array}$ & $\begin{array}{c}5.11 \\
(2.11)\end{array}$ & $\begin{array}{c}5.95 \\
(2.42)\end{array}$ & $\begin{array}{c}6.08 \\
(2.45)\end{array}$ & $\begin{array}{c}5.99 \\
(2.43)\end{array}$ & $\begin{array}{c}5.75 \\
(3.38)\end{array}$ & $\begin{array}{c}5.63 \\
(2.35)\end{array}$ & $\begin{array}{c}5.09 \\
(2.23)\end{array}$ & $\begin{array}{c}5.21 \\
(2.25)\end{array}$ & $\begin{array}{c}4.82 \\
(2.16)\end{array}$ & $\begin{array}{c}4.11 \\
(1.98)\end{array}$ & $\begin{array}{c}3.88 \\
(1.92)\end{array}$ & $\begin{array}{c}3.41 \\
(1.78)\end{array}$ & $\begin{array}{c}3.24 \\
(1.73)\end{array}$ \\
\hline Quinalphos 25\% EC & 375 & $\begin{array}{c}7.05 \\
(2.65)\end{array}$ & $\begin{array}{c}6.97 \\
(2.63)\end{array}$ & $\begin{array}{c}5.36 \\
(2.29)\end{array}$ & $\begin{array}{c}5.13 \\
(2.24)\end{array}$ & $\begin{array}{c}5.56 \\
(2.33)\end{array}$ & $\begin{array}{c}5.72 \\
(2.37)\end{array}$ & $\begin{array}{c}5.52 \\
(2.33)\end{array}$ & $\begin{array}{c}5.19 \\
(2.25)\end{array}$ & $\begin{array}{c}5.07 \\
(2.22)\end{array}$ & $\begin{array}{c}4.35 \\
(2.04)\end{array}$ & $\begin{array}{c}4.39 \\
(2.05)\end{array}$ & $\begin{array}{c}4.19 \\
(2.00)\end{array}$ & $\begin{array}{c}4.07 \\
(1.97)\end{array}$ & $\begin{array}{c}3.71 \\
(1.87)\end{array}$ & $\begin{array}{c}3.32 \\
(1.75)\end{array}$ & $\begin{array}{c}3.18 \\
(1.71)\end{array}$ \\
\hline Quinalphos 25\% EC & 750 & $\begin{array}{c}6.78 \\
(2.59)\end{array}$ & $\begin{array}{c}6.64 \\
(2.57)\end{array}$ & $\begin{array}{c}5.05 \\
(2.22)\end{array}$ & $\begin{array}{c}4.85 \\
(2.19)\end{array}$ & $\begin{array}{c}5.05 \\
(2.22)\end{array}$ & $\begin{array}{c}5.14 \\
(2.24)\end{array}$ & $\begin{array}{c}5.03 \\
(2.21)\end{array}$ & $\begin{array}{c}4.73 \\
(2.14)\end{array}$ & $\begin{array}{c}4.62 \\
(2.11)\end{array}$ & $\begin{array}{c}4.02 \\
(1.96)\end{array}$ & $\begin{array}{c}4.19 \\
(2.00)\end{array}$ & $\begin{array}{c}3.87 \\
(1.92)\end{array}$ & $\begin{array}{c}3.19 \\
(1.71)\end{array}$ & $\begin{array}{c}2.53 \\
(1.50)\end{array}$ & $\begin{array}{c}2.28 \\
(1.40)\end{array}$ & $\begin{array}{c}2.08 \\
(1.32)\end{array}$ \\
\hline $\begin{array}{l}\text { Indoxacarb } 14.5 \% \mathrm{SC} \\
\text { (Standard treatment) }\end{array}$ & 60 & $\begin{array}{c}6.94 \\
(2.63)\end{array}$ & $\begin{array}{c}6.81 \\
(2.60)\end{array}$ & $\begin{array}{c}5.94 \\
(2.42)\end{array}$ & $\begin{array}{l}5.85 \\
(2.40)\end{array}$ & $\begin{array}{c}5.97 \\
(2.43)\end{array}$ & $\begin{array}{c}6.08 \\
(2.45)\end{array}$ & $\begin{array}{c}5.83 \\
(2.39)\end{array}$ & $\begin{array}{c}5.58 \\
(2.34)\end{array}$ & $\begin{array}{l}5.45 \\
(2.31)\end{array}$ & $\begin{array}{c}5.02 \\
(2.21)\end{array}$ & $\begin{array}{c}5.17 \\
(2.25)\end{array}$ & $\begin{array}{c}4.91 \\
(2.18)\end{array}$ & $\begin{array}{c}4.15 \\
(1.99)\end{array}$ & $\begin{array}{c}3.78 \\
(1.89)\end{array}$ & $\begin{array}{c}3.46 \\
(1.80)\end{array}$ & $\begin{array}{l}3.28 \\
(1.74)\end{array}$ \\
\hline $\begin{array}{l}\text { Deltamethrin } 2.8 \% \text { EC } \\
\text { (Standard treatment) }\end{array}$ & 12.5 & $\begin{array}{c}6.88 \\
(2.61)\end{array}$ & $\begin{array}{c}6.78 \\
(2.59)\end{array}$ & $\begin{array}{c}6.51 \\
(2.54)\end{array}$ & $\begin{array}{c}6.47 \\
(2.53)\end{array}$ & $\begin{array}{c}6.57 \\
(2.55)\end{array}$ & $\begin{array}{c}6.63 \\
(2.56)\end{array}$ & $\begin{array}{c}6.45 \\
(2.53)\end{array}$ & $\begin{array}{c}6.23 \\
(2.48)\end{array}$ & $\begin{array}{c}6.12 \\
(2.46)\end{array}$ & $\begin{array}{c}5.79 \\
(2.78)\end{array}$ & $\begin{array}{c}5.85 \\
(2.39)\end{array}$ & $\begin{array}{c}5.55 \\
(2.33)\end{array}$ & $\begin{array}{c}5.14 \\
(2.24)\end{array}$ & $\begin{array}{c}4.71 \\
(2.13)\end{array}$ & $\begin{array}{c}4.53 \\
(2.09)\end{array}$ & $\begin{array}{l}4.30 \\
(2.03)\end{array}$ \\
\hline Untreated control & - & $\begin{array}{l}7.37 \\
(2.71)\end{array}$ & $\begin{array}{l}7.09 \\
(2.66)\end{array}$ & $\begin{array}{c}7.14 \\
(2.67)\end{array}$ & $\begin{array}{c}7.30 \\
(2.70)\end{array}$ & $\begin{array}{c}7.41 \\
(2.72)\end{array}$ & $\begin{array}{l}7.55 \\
(2.74)\end{array}$ & $\begin{array}{c}7.72 \\
(2.78)\end{array}$ & $\begin{array}{c}7.84 \\
(2.80)\end{array}$ & $\begin{array}{c}8.01 \\
(2.83)\end{array}$ & $\begin{array}{c}8.30 \\
(2.88)\end{array}$ & $\begin{array}{l}8.37 \\
(2.90)\end{array}$ & $\begin{array}{c}8.64 \\
(2.94)\end{array}$ & $\begin{array}{c}8.84 \\
(2.98)\end{array}$ & $\begin{array}{c}8.92 \\
(2.99)\end{array}$ & $\begin{array}{c}9.00 \\
(3.01)\end{array}$ & $\begin{array}{c}9.00 \\
(3.01)\end{array}$ \\
\hline $\mathrm{CD}$ at $5 \%$ & & NS & 0.05 & 0.05 & 0.05 & 0.08 & 0.03 & 0.07 & 0.04 & 0.05 & 0.03 & 0.04 & 0.07 & 0.07 & 0.07 & 0.06 & 0.06 \\
\hline
\end{tabular}

Figures in the parentheses are $\sqrt{\mathrm{x}}+0.5$ transformation; PTC - Pre Treatment Count; DAS - Days After Spraying

Table.2 Bio-efficacy of Quinalphos 25\% EC against H. armigera on red gram during Kharif, 2017

\begin{tabular}{|c|c|c|c|c|c|c|c|c|c|c|c|c|c|c|c|c|c|}
\hline \multirow{3}{*}{ Treatments } & \multirow{3}{*}{$\begin{array}{c}\text { Dose } \\
\text { (g a.i./ha) }\end{array}$} & \multirow{3}{*}{ PTC } & \multicolumn{15}{|c|}{ Average no. of larval population / 5 Plants } \\
\hline & & & \multicolumn{5}{|c|}{ First Spray } & \multicolumn{5}{|c|}{ Second spray } & \multicolumn{5}{|c|}{ Third spray } \\
\hline & & & 1 DAS & $\begin{array}{c}3 \\
\text { DASS }\end{array}$ & 5 DAS & 7 DAS & $\begin{array}{c}10 \\
\text { DAS }\end{array}$ & 1 DAS & 3 DAS & 5 DAS & 7 DAS & $\begin{array}{c}\text { 10 } \\
\text { DAS }\end{array}$ & 1 DAS & 3 DAS & 5 DAS & $\begin{array}{c}7 \\
\text { DAS }\end{array}$ & $\begin{array}{c}10 \\
\text { DAS }\end{array}$ \\
\hline Quinalphos 25\% EC & 325 & $\begin{array}{c}6.32 \\
(3.01)\end{array}$ & $\begin{array}{c}6.16 \\
(2.98)\end{array}$ & $\begin{array}{c}5.87 \\
(2.92)\end{array}$ & $\begin{array}{c}5.83 \\
(2.91)\end{array}$ & $\begin{array}{c}5.98 \\
(2.95)\end{array}$ & $\begin{array}{c}6.04 \\
(2.96)\end{array}$ & $\begin{array}{c}5.71 \\
(2.89)\end{array}$ & $\begin{array}{c}5.53 \\
(2.85)\end{array}$ & $\begin{array}{c}5.44 \\
(2.83)\end{array}$ & $\begin{array}{c}4.72 \\
(2.67)\end{array}$ & $\begin{array}{c}4.80 \\
(2.69)\end{array}$ & $\begin{array}{c}4.57 \\
(2.64)\end{array}$ & $\begin{array}{c}4.34 \\
(2.53)\end{array}$ & $\begin{array}{c}3.69 \\
(2.42)\end{array}$ & $\begin{array}{c}3.34 \\
(2.33)\end{array}$ & $\begin{array}{c}3.09 \\
(2.26)\end{array}$ \\
\hline Quinalphos 25\% EC & 350 & $\begin{array}{c}6.13 \\
(2.98)\end{array}$ & $\begin{array}{c}6.07 \\
(2.96)\end{array}$ & $\begin{array}{c}4.87 \\
(2.71)\end{array}$ & $\begin{array}{c}4.50 \\
(2.61)\end{array}$ & $\begin{array}{c}5.34 \\
(2.81)\end{array}$ & $\begin{array}{c}5.47 \\
(2.84)\end{array}$ & $\begin{array}{c}5.38 \\
(2.82)\end{array}$ & $\begin{array}{c}5.14 \\
(2.77)\end{array}$ & $\begin{array}{c}5.02 \\
(2.74)\end{array}$ & $\begin{array}{c}4.48 \\
(2.62)\end{array}$ & $\begin{array}{l}4.60 \\
(2.64)\end{array}$ & $\begin{array}{c}4.21 \\
(2.55)\end{array}$ & $\begin{array}{c}3.50 \\
(2.37)\end{array}$ & $\begin{array}{c}3.27 \\
(2.30)\end{array}$ & $\begin{array}{c}2.80 \\
(2.17)\end{array}$ & $\begin{array}{c}2.63 \\
(2.12)\end{array}$ \\
\hline Quinalphos 25\% EC & 375 & $\begin{array}{c}6.44 \\
(3.04)\end{array}$ & $\begin{array}{c}6.36 \\
(3.02)\end{array}$ & $\begin{array}{c}4.75 \\
(2.68)\end{array}$ & $\begin{array}{c}4.52 \\
(2.63)\end{array}$ & $\begin{array}{c}4.95 \\
(2.72)\end{array}$ & $\begin{array}{c}5.11 \\
(2.76)\end{array}$ & $\begin{array}{c}4.91 \\
(2.72)\end{array}$ & $\begin{array}{c}4.85 \\
(2.64)\end{array}$ & $\begin{array}{c}4.46 \\
(2.61)\end{array}$ & $\begin{array}{c}3.74 \\
(2.43)\end{array}$ & $\begin{array}{c}2.78 \\
(2.44)\end{array}$ & $\begin{array}{c}3.58 \\
(2.39)\end{array}$ & $\begin{array}{c}3.46 \\
(2.36)\end{array}$ & $\begin{array}{c}3.10 \\
(2.26)\end{array}$ & $\begin{array}{c}2.71 \\
(2.14)\end{array}$ & $\begin{array}{c}2.57 \\
(2.10)\end{array}$ \\
\hline Quinalphos 25\% EC & 750 & $\begin{array}{c}6.17 \\
(2.98)\end{array}$ & $\begin{array}{c}6.03 \\
(2.96)\end{array}$ & $\begin{array}{c}4.44 \\
(2.61)\end{array}$ & $\begin{array}{c}4.34 \\
(2.58)\end{array}$ & $\begin{array}{c}4.44 \\
(2.61)\end{array}$ & $\begin{array}{c}4.53 \\
(2.63)\end{array}$ & $\begin{array}{c}4.42 \\
(2.60)\end{array}$ & $\begin{array}{c}4.12 \\
(2.53)\end{array}$ & $\begin{array}{c}4.01 \\
(2.50)\end{array}$ & $\begin{array}{c}3.41 \\
(2.35)\end{array}$ & $\begin{array}{c}3.58 \\
(2.39)\end{array}$ & $\begin{array}{c}3.26 \\
(2.31)\end{array}$ & $\begin{array}{c}2.58 \\
(2.10)\end{array}$ & $\begin{array}{c}1.92 \\
(1.89)\end{array}$ & $\begin{array}{c}1.67 \\
(1.79)\end{array}$ & $\begin{array}{c}1.47 \\
(1.71)\end{array}$ \\
\hline $\begin{array}{l}\text { Indoxacarb } 14.5 \% \mathrm{SC} \\
\text { (Standard treatment) }\end{array}$ & 60 & $\begin{array}{c}6.33 \\
(3.02)\end{array}$ & $\begin{array}{c}6.20 \\
(2.99)\end{array}$ & $\begin{array}{c}5.33 \\
(2.81)\end{array}$ & $\begin{array}{c}5.24 \\
(2.79)\end{array}$ & $\begin{array}{c}5.36 \\
(2.82)\end{array}$ & $\begin{array}{l}5.47 \\
(2.84)\end{array}$ & $\begin{array}{c}5.22 \\
(2.78)\end{array}$ & $\begin{array}{l}4.97 \\
(2.73)\end{array}$ & $\begin{array}{c}4.84 \\
(2.70)\end{array}$ & $\begin{array}{c}4.41 \\
(2.60)\end{array}$ & $\begin{array}{c}4.56 \\
(2.64)\end{array}$ & $\begin{array}{l}4.30 \\
(2.57)\end{array}$ & $\begin{array}{c}3.54 \\
(2.38)\end{array}$ & $\begin{array}{c}3.17 \\
(2.28)\end{array}$ & $\begin{array}{l}2.85 \\
(2.19)\end{array}$ & $\begin{aligned} 2.67 \\
(2.13)\end{aligned}$ \\
\hline $\begin{array}{l}\text { Deltamethrin } 2.8 \% \text { EC } \\
\text { (Standard treatment) }\end{array}$ & 12.5 & $\begin{array}{c}6.27 \\
(3.00)\end{array}$ & $\begin{array}{c}6.17 \\
(2.98)\end{array}$ & $\begin{array}{c}5.90 \\
(2.93)\end{array}$ & $\begin{array}{c}5.86 \\
(2.92)\end{array}$ & $\begin{array}{c}5.96 \\
(2.94)\end{array}$ & $\begin{array}{c}6.02 \\
(2.95)\end{array}$ & $\begin{array}{c}5.84 \\
(2.92)\end{array}$ & $\begin{array}{c}5.62 \\
(2.87)\end{array}$ & $\begin{array}{c}5.51 \\
(2.85)\end{array}$ & $\begin{array}{c}5.18 \\
(3.17)\end{array}$ & $\begin{array}{c}5.24 \\
(2.78)\end{array}$ & $\begin{array}{c}4.94 \\
(2.72)\end{array}$ & $\begin{array}{c}4.53 \\
(2.63)\end{array}$ & $\begin{array}{c}4.10 \\
(2.52)\end{array}$ & $\begin{array}{c}3.92 \\
(2.48)\end{array}$ & $\begin{array}{r}3.69 \\
(2.42)\end{array}$ \\
\hline Untreated control & - & $\begin{array}{c}6.76 \\
(3.19)\end{array}$ & $\begin{array}{c}6.48 \\
(3.05)\end{array}$ & $\begin{array}{c}6.53 \\
(3.06)\end{array}$ & $\begin{array}{c}6.69 \\
(3.09)\end{array}$ & $\begin{array}{c}6.80 \\
(3.11)\end{array}$ & $\begin{array}{c}6.94 \\
(3.13)\end{array}$ & $\begin{array}{c}7.11 \\
(3.17)\end{array}$ & $\begin{array}{l}7.23 \\
(3.19)\end{array}$ & $\begin{array}{c}7.40 \\
(3.22)\end{array}$ & $\begin{array}{c}7.69 \\
(3.27)\end{array}$ & $\begin{array}{c}7.76 \\
(3.29)\end{array}$ & $\begin{array}{c}8.03 \\
(3.33)\end{array}$ & $\begin{array}{c}8.23 \\
(2.37)\end{array}$ & $\begin{array}{c}8.31 \\
(3.38)\end{array}$ & $\begin{array}{c}8.39 \\
(3.40)\end{array}$ & $\begin{array}{c}8.50 \\
(3.41)\end{array}$ \\
\hline $\mathrm{CD}$ at $5 \%$ & & NS & 0.03 & 0.03 & 0.03 & 0.06 & 0.01 & 0.05 & 0.02 & 0.03 & 0.02 & 0.03 & 0.05 & 0.05 & 0.05 & 0.04 & 0.04 \\
\hline
\end{tabular}

Figures in the parentheses are $\sqrt{\mathrm{x}}+0.5$ transformation; PTC - Pre Treatment Count; DAS - Days After Spraying 
Table.3 Effect of different doses of Quinalphos 25\% EC on per cent pod damage by pod borer, $H$. armigera

\begin{tabular}{|c|l|c|c|c|}
\hline \multirow{2}{*}{$\begin{array}{c}\text { S. } \\
\text { No }\end{array}$} & \multicolumn{1}{|c|}{ Treatments } & $\begin{array}{c}\text { Dose } \\
\text { (g a.i./ha) }\end{array}$ & \multicolumn{2}{|c|}{ **Pod damage (\%) by } \\
\cline { 3 - 5 } & & & $\mathbf{2 0 1 6}$ & $\mathbf{2 0 1 7}$ \\
\hline 1. & Quinalphos 25\% EC & 325 & $8.09(16.34)$ & $10.05(17.58)$ \\
\hline 2. & Quinalphos 25\% EC & 350 & $7.20(15.38)$ & $9.18(16.60)$ \\
\hline 3. & Quinalphos 25\% EC & 375 & $6.91(15.04)$ & $8.89(16.26)$ \\
\hline 4. & Quinalphos 25\% EC & 750 & $4.99(12.45)$ & $6.97(13.67)$ \\
\hline $\mathbf{5 .}$ & $\begin{array}{l}\text { Indoxacarb 14.5\% SC } \\
\text { (Standard treatment) }\end{array}$ & 60 & $7.76(16.05)$ & $9.74(17.27)$ \\
\hline 6. & $\begin{array}{l}\text { Deltamethrin 2.8\% EC } \\
\text { (Standard treatment) }\end{array}$ & 12.5 & $8.10(16.44)$ & $10.08(17.66)$ \\
\hline 7. & Untreated control & - & $10.96(19.40)$ & $12.94(20.62)$ \\
\hline & CD at 5\% & & $\mathbf{1 . 2 7}$ & $\mathbf{1 . 3 0}$ \\
\hline
\end{tabular}

${ }^{* *}$ Figures in the parentheses are Angular transformed values

Table.4 Effect of different doses of Quinalphos 25\% EC on per cent pod damage by pod fly, M. obtusa

\begin{tabular}{|c|c|c|c|c|}
\hline \multirow[t]{2}{*}{ S.No } & \multirow[t]{2}{*}{ Treatments } & \multirow[t]{2}{*}{$\begin{array}{c}\text { Dose } \\
\text { (g a.i./ha) }\end{array}$} & \multicolumn{2}{|c|}{$\begin{array}{c}\text { **Pod damage }(\%) \text { by } M . \\
\text { obtuse }\end{array}$} \\
\hline & & & 2016 & 2017 \\
\hline 1. & Quinalphos $25 \%$ EC & 325 & $35.30(35.56)$ & $39.80(39.96)$ \\
\hline 2. & Quinalphos 25\% EC & 350 & $33.83(34.69)$ & $38.13(38.99)$ \\
\hline 3. & Quinalphos 25\% EC & 375 & $33.41(34.43)$ & $37.71(38.73)$ \\
\hline 4. & Quinalphos 25\% EC & 750 & $33.04(34.22)$ & $37.34(38.52)$ \\
\hline 5. & $\begin{array}{l}\text { Indoxacarb } 14.5 \% \mathrm{SC} \\
\text { (Standard treatment) }\end{array}$ & 60 & $34.21(34.92)$ & $38.51(39.22)$ \\
\hline 6. & $\begin{array}{l}\text { Deltamethrin } 2.8 \% \text { EC } \\
\text { (Standard treatment) }\end{array}$ & 12.5 & $35.47(35.72)$ & $39.87(40.02)$ \\
\hline 7. & Untreated control & - & $40.39(38.96)$ & $43.69(42.26)$ \\
\hline \multicolumn{3}{|c|}{ CD at $5 \%$} & 1.06 & 1.11 \\
\hline
\end{tabular}

**Figures in the parentheses are Angular transformed values 


\section{References}

Armes, N. J., Jadhav, D. R., Bond, G. S. and King, A. B. S., 1992. Insecticide resistance in Helicoverpa armigera in South India. Pestic. Sci. 34: 355-364.

Bhubaneshwari, K. and Balasuranathan L., 2002. Pod borer complex of pigeon pea in Tamilnadu. Insect Environment 8: 160-161

Lal, S. S., 1981. Needs and priorities for developing integrated pest management for pigeon pea pest. Pulse Crop News Letter 1: 48-49.

Lal, S. S. Yadav, C. P. and Ahmad, R., 1997. Insect pests of short duration pigeonpea- A review. Plant Prot. Bull. (Faridabad) 49:25-32.

Mccafferry, A. K., King, A. B. S., Walker, A. J. and Nayir, H., 1989. Resistance to synthetic pyrethrroides in the bollworm Helicoverpa armigera from Andhra Pradesh, India Pestic. Sci. 27: 65-76.

Panda, B. C., 2005. Crop production and management Agronomy. Fertiliser Statistics: 449-450.

Pawar, A. D. and Bhalla, O. P., 1975. Occurrence of $\mathrm{H}$. armigera as a serious pest of arhar in Himachal Pradesh. International Pigeon Pea News Letter 5: 24.

Prasad, C. S., 1997. Host range and seasonal incidence of Helicoverpa armigera in the lower Kumaon hills of U.P. Insect Environment 3: 33-34.

Sanap, M. M., Aher, R. P. and Deshmukh, R.
B., 1995. Incidence of major insect pests associated with pigeonpea at Rahuri, India. Indian J. Pulses Research 8: 192-194

Singh, H. M., Singh, R. and Rizvi, S. M. A., 1988. Insecticidal evaluation against pigeon pea pod borers. Indian J. Plant. Prot. 16: 217-220

Singh, R. H. and Singh, K. M., 1978. Succession of inset pests in early varieties of red gram [Cajanus cajan (L.) Mill spp.] Indian J. Ent. 40: 1-6.

Sithanantham, S., Lateef, S. S. and Reed, W. 1987. Pigeonpea insect identification hand book. Information Bull. 26 ICRISAT Patancheru, Andhra Pradesh, pp: 1 - 26

Sunil, Kumar, B. Singh and Neeraj Kumar 2003. Assessment of pod damage caused by pod borer complex in pre rabi pigeonpea. Indian J. Pulses Research 16: 169-170

Singh, H.K and Singh, H. N., 1991. Some major pest incidence on certain late cultivars of pigeonpea, Cajanus cajan L. during pod formation stage. Indian $\mathrm{J}$. Ent. 53: 298-303.

Thakur, R. C., Nema, K. K. and Singh O. P., 1989. Losses caused by pod fly (Melanagromyza obtusa Mall) and pod borer [Helicoverpa armigera (Hub)] to pigeonpea in Madhya Pradesh. Bhartiya Krishi Anusandhan Patrika 4: 107-111.

\section{How to cite this article:}

Vyas, A., M.K. Mahla, G. Chhangani and Dangi, N.L. 2018. Bio-efficacy Studies of Quinalphos 25\% EC against Insect Pests of Red Gram. Int.J.Curr.Microbiol.App.Sci. 7(07): 601-606. doi: https://doi.org/10.20546/ijcmas.2018.707.073 